\title{
Video Article \\ An Atmospheric Pressure Plasma Setup to Investigate the Reactive Species Formation
}

\author{
Yury Gorbanev ${ }^{1,2}$, Robert Soriano ${ }^{2}$, Deborah O'Connell ${ }^{2}$, Victor Chechik ${ }^{1}$ \\ ${ }^{1}$ Department of Chemistry, University of York \\ ${ }^{2}$ York Plasma Institute, Department of Physics, University of York
}

Correspondence to: Victor Chechik at victor.chechik@york.ac.uk

URL: https://www.jove.com/video/54765

DOI: doi: $10.3791 / 54765$

Keywords: Engineering, Issue 117, cold plasma, atmospheric pressure plasma jet, reactive species, controlled atmosphere reactor, plasma effluent, Faraday cage, radical spin trapping, isotopically labelled water, physics

Date Published: 11/3/2016

Citation: Gorbanev, Y., Soriano, R., O'Connell, D., Chechik, V. An Atmospheric Pressure Plasma Setup to Investigate the Reactive Species Formation. J. Vis. Exp. (117), e54765, doi:10.3791/54765 (2016).

\section{Abstract}

Non-thermal atmospheric pressure ('cold') plasmas have received increased attention in recent years due to their significant biomedical potential. The reactions of cold plasma with the surrounding atmosphere yield a variety of reactive species, which can define its effectiveness. While efficient development of cold plasma therapy requires kinetic models, model benchmarking needs empirical data. Experimental studies of the source of reactive species detected in aqueous solutions exposed to plasma are still scarce. Biomedical plasma is often operated with He or $\mathrm{Ar}$ feed gas, and a specific interest lies in investigation of the reactive species generated by plasma with various gas admixtures $\left(\mathrm{O}_{2}, \mathrm{~N}_{2}\right.$, air, $\mathrm{H}_{2} \mathrm{O}$ vapor, etc.) Such investigations are very complex due to difficulties in controlling the ambient atmosphere in contact with the plasma effluent. In this work, we addressed common issues of 'high' voltage $\mathrm{kHz}$ frequency driven plasma jet experimental studies. A reactor was developed allowing the exclusion of ambient atmosphere from the plasma-liquid system. The system thus comprised the feed gas with admixtures and the components of the liquid sample. This controlled atmosphere allowed the investigation of the source of the reactive oxygen species induced in aqueous solutions by He-water vapor plasma. The use of isotopically labelled water allowed distinguishing between the species originating in the gas phase and those formed in the liquid. The plasma equipment was contained inside a Faraday cage to eliminate possible influence of any external field. The setup is versatile and can aid in further understanding the cold plasma-liquid interactions chemistry.

\section{Video Link}

The video component of this article can be found at https://www.jove.com/video/54765/

\section{Introduction}

Low-temperature atmospheric pressure plasmas (LTPs) have attracted increased attention in recent years due to their vast potential for biomedical applications ${ }^{1-3}$. Upon contact with ambient atmosphere, LTP reacts with molecular content of air $\left(\mathrm{N}_{2}, \mathrm{O}_{2}, \mathrm{H}_{2} \mathrm{O}\right.$ vapor), generating a variety of reactive oxygen and nitrogen species (RONS) ${ }^{2,4}$. Among these are relatively stable species (such as hydrogen peroxide, ozone, nitrite and nitrate anions) and highly reactive radicals $\left(\cdot \mathrm{OH}, \cdot \mathrm{OOH} / \mathrm{O}_{2} \cdot{ }^{-} \cdot \bullet \mathrm{H}, \cdot \mathrm{NO}\right.$, etc.). These species, initially generated in the gas phase, are further delivered by the plasma to the biological substrate ${ }^{5}$. RONS interact with substrates and thus define the antimicrobial, anticancer and antiviral effects of LTP ${ }^{6-8}$

The development of LTP therapies requires complex modelling of the reactions of RONS ${ }^{9}$. Water is an essential part of the biological milieu, and the reactions in the aqueous phase increase the complexity of the system dramatically. The investigation of the gas-phase plasma is widely performed using various analytical techniques, including optical emission spectroscopy, laser induced fluorescence, infrared spectroscopy, mass spectrometry (MS), etc. ${ }^{10-12}$. At the same time, detailed investigations of the species detected in the liquid phase are still scarce. Available reports describe the use of various analytical methods such as UV and electron paramagnetic resonance (EPR) spectroscopy, cytometry, etc. for the detection of RONS in aqueous solutions ${ }^{13,14}$. EPR is one of the most direct methods for radical detection in the liquid. However, many radical species cannot be detected by EPR due to their short life time. In these cases, spin trapping is often used. Spin trapping a technique involving a compound (spin trap) which rapidly and selectively reacts with the radical to yield a more persistent radical adduct (e.g., DMPO reacts with the hydroxyl radical, forming DMPO-OH adduct).

The common challenges in plasma-liquid interaction studies are the inability to control the ambient atmosphere around the plasma effluent and other interfering factors (external fields, environment-sensitive power supply parts, etc.). Here, we demonstrate the use of a setup comprising of a metal mesh case containing the operated plasma and an in-house built reactor around the plasma jet nozzle. The metal mesh serves as the Faraday cage, allowing significantly improved reproducibility and general operability of the plasma jet. The glass reactor encapsulates both the plasma jet and the liquid sample, excluding the surrounding atmosphere from the system. 
This method can be employed for any atmospheric pressure plasma jet in contact with liquid solutions. For example, we have recently presented an investigation of the source of the reactive oxygen species detected in an aqueous sample exposed to plasma. Isotopically labelled water was used to distinguish between the species formed in the liquid and in the gas phase of the plasma jet-liquid solution system ${ }^{15}$.

\section{Protocol}

\section{Shielding the Plasma Setup}

1. Position all parts of the electric environment inside the cage: power supply, voltage/current meter, power cable, plasma electrodes, plasma jet, etc.

2. Make sure that the space within the cage is sufficient so that the live electrode, the ground electrode and respective cables are not in contact with each other or the metal mesh.

3. Equip the cage with an interlock connected to the plasma power supply to avoid the risk of electric shock from the high voltage electrode during the plasma operation.

4. Place the voltage and frequency controls on the outer surface of the cage to allow changing of the parameters without interrupting the plasma operation.

5. Ground all metal supports inside the mesh cage and the cage itself by wiring them to an earth bonding plug.

\section{Discharge Parameters}

1. Position the live electrode below the ground electrode on the glass tube (i.e., closer to the tube nozzle).

2. Connect the voltage probe to the plasma power supply to measure the operating voltage, and pass the ground electrode through the circular current probe to monitor the return current.

3. Connect both the voltage and the current probe to an oscilloscope, monitoring the current, the voltage and the plasma operating frequency (determined by either the current or the voltage probe).

4. Set the flow of gas through the glass tube to 2 slm using the mass flow controllers (MFCs).

5. Ignite the plasma in a glass tube with the feed gas helium passing through it by turning on the plasma power supply. Using the readings from the probes, set the voltage and frequency of the discharge to $18 \mathrm{kV}$ and $25 \mathrm{kHz}$, respectively. NOTE: Parameter variations are carried out to determine the minimal voltage and frequency at which the discharge is occurring with the highest molecular content of all the experiments. The increased molecular content requires higher voltage for the plasma to be ignited. Note that elevated voltages can result in significant gas temperature increase of the plasma, thus leading to increased evaporation of the liquid sample.

6. Keep the voltage constant in all experiments.

\section{Introducing Admixtures to the Feed Gas}

1. Connect the second MFC to the main feed gas tubing using a T-connector.

2. To add the water vapor to the feed gas, direct an MFC-regulated flow of helium through a Drechsel flask filled with water and positioned outside (on top or on the side) of the mesh cage.

3. Obtain a desired level of saturation by splitting the flow of the feed gas. Direct $10 \%$ of the gas flow $(200 \mathrm{sccm})$ through the Drechsel flask with water $\left(\mathrm{H}_{2}{ }^{16} \mathrm{O}\right)$ to achieve a $10 \%$ saturation of the feed gas.

4. Using the T-connector, combine this fully water vapor-saturated gas with the $90 \%(1,800 \mathrm{sccm})$ of the dry gas flow.

\section{The Reactor}

1. Prepare a glass reactor consisting of two parts, upper and lower. Equip the lower part of the with an exhaust tube.

2. Position the glass reactor at the nozzle of the plasma jet.

3. Insert the plasma jet nozzle into a rubber grommet inside the opening in the upper part of the reactor.

4. Prepare the container consisting of a well-like reservoir on top of a stand. Make both the stand and the well from a dielectric material (e.g., glass, quartz glass).

5. Place the sample container inside the reactor so that it is exposed to the plasma effluent from the nozzle of the jet.

6. Put a liquid $\mathrm{H}_{2}{ }^{17} \mathrm{O}$ sample inside the sample container. For the detection of the hydroxyl radicals, use a solution of the 5,5-dimethyl-1pyrroline- $N$-oxide (DMPO) spin trap (see 5.1).

NOTE: The choice of the spin trap as well as the choice of the liquid sample components depends on the specific species investigated. For example, the source of the $\cdot \mathrm{OH}$ radical is studied using $\mathrm{H}_{2}{ }^{16} \mathrm{O} / \mathrm{H}_{2}{ }^{17} \mathrm{O}$ and DMPO spin trap. The source of $\bullet \mathrm{H}$ radical requires the use of an $\mathrm{H}_{2} \mathrm{O} / \mathrm{D}_{2} \mathrm{O}$ (gas and liquid). $\mathrm{N}$-tert-Butyl-a-phenylnitrone (PBN) should be used for the detection of the $\bullet \mathrm{H}$ radical. In the case of $\mathrm{He}$ plasma with $\mathrm{H}_{2} \mathrm{O}$ vapor, it was shown to predominantly trap hydrogen radical, while DMPO formed mostly DMPO-OH adduct ${ }^{15}$.

7. Connect the two reactor parts via the ground glass surfaces contact.

\section{Spin Trapping of Radical Species}

1. Prepare the solutions of the chosen spin trap with the required concentration. For aqueous solutions, use de-ionized water. For nitrone spin traps (such as DMPO), use a $100 \mathrm{mM}$ concentration.

2. Pre-flush the reactor with the feed gas $(2 \mathrm{slm})$ for $30 \mathrm{sec}$

3. Ignite the plasma (see 2.5) and expose the liquid sample to the plasma effluent for a set period of time (e.g., $60 \mathrm{sec}$ ).

4. After a required exposure time, switch off the plasma power supply and open the reactor. Remove the sample container from the reactor 
5. Collect the sample and analyze it using the electron paramagnetic resonance spectroscopy (EPR $)^{15}$.

\section{Representative Results}

Using the method and equipment described above, we have investigated the origin of the reactive oxygen species in the LTP system in contact with water. The plasma operating frequency and voltage were $25 \mathrm{kHz}$ and $18 \mathrm{kV}$ (peak-to-peak), respectively (Figure 1).

For example, the source of the hydroxyl radical was determined using the isotopically labelled water. This enabled distinguishing between the water molecules in the feed gas from those in the liquid sample. For that, $\mathrm{H}_{2}{ }^{16} \mathrm{O}$ was introduced in the feed gas (as vapor). A liquid sample of $\mathrm{H}_{2}{ }^{17} \mathrm{O}$ with the dissolved spin trap DMPO was positioned in a sample container. The reactor was pre-flushed for 30 sec with the feed gas. Importantly, in this case longer pre-flushing time may result in substantial amount of $\mathrm{H}_{2}{ }^{16} \mathrm{O}$ delivered to the liquid $\mathrm{H}_{2}{ }^{17} \mathrm{O}$ sample. Then, the plasma was ignited and the sample was exposed to the effluent for $60 \mathrm{sec}$. The post-exposure solution was analyzed by EPR. Two DMPO-OH radical adducts (DMPO- ${ }^{17} \mathrm{OH}$ and DMPO- ${ }^{16} \mathrm{OH}$ ) were detected (Figure 2). The ratio of the adducts formed was determined via further analysis of the EPR data. The MS analysis of the liquid phase composition showed the ratio of $\mathrm{H}_{2}{ }^{16} \mathrm{O}$ (diffused into the liquid from the gas phase) to $\mathrm{H}_{2}{ }^{17} \mathrm{O}$ (Table 1). The comparison of the two suggested that the hydroxyl radicals detected in the liquid were, in fact, originating in the gas phase, and not in the liquid.

Similar studies can be performed by using other systems, such as a $\mathrm{D}_{2} \mathrm{O} / \mathrm{H}_{2} \mathrm{O}$ system to detect the source of the $\cdot H(\cdot \mathrm{D})$ radical ${ }^{15}$.

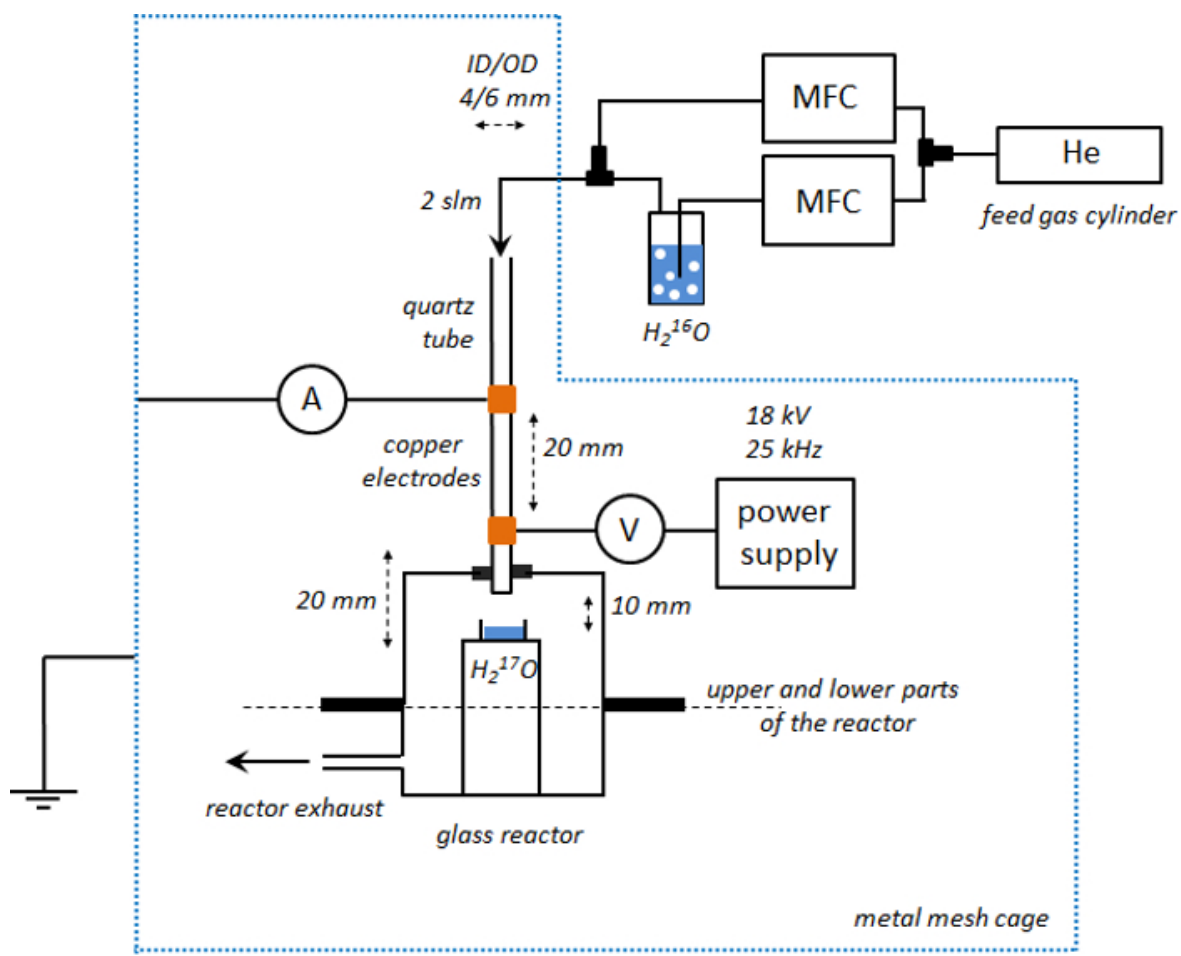

Figure 1. Setup used for the investigation of the source of reactive oxygen species. The plasma was generated in a quartz glass tube ( $4 \mathrm{~mm}$ internal diameter, $1 \mathrm{~mm}$ wall thickness) with the helium feed gas. The feed gas flow was $2 \mathrm{slm}$. The feed gas contained $\mathrm{H}_{2} \mathrm{O}$ vapor introduced as stated above. Please click here to view a larger version of this figure. 

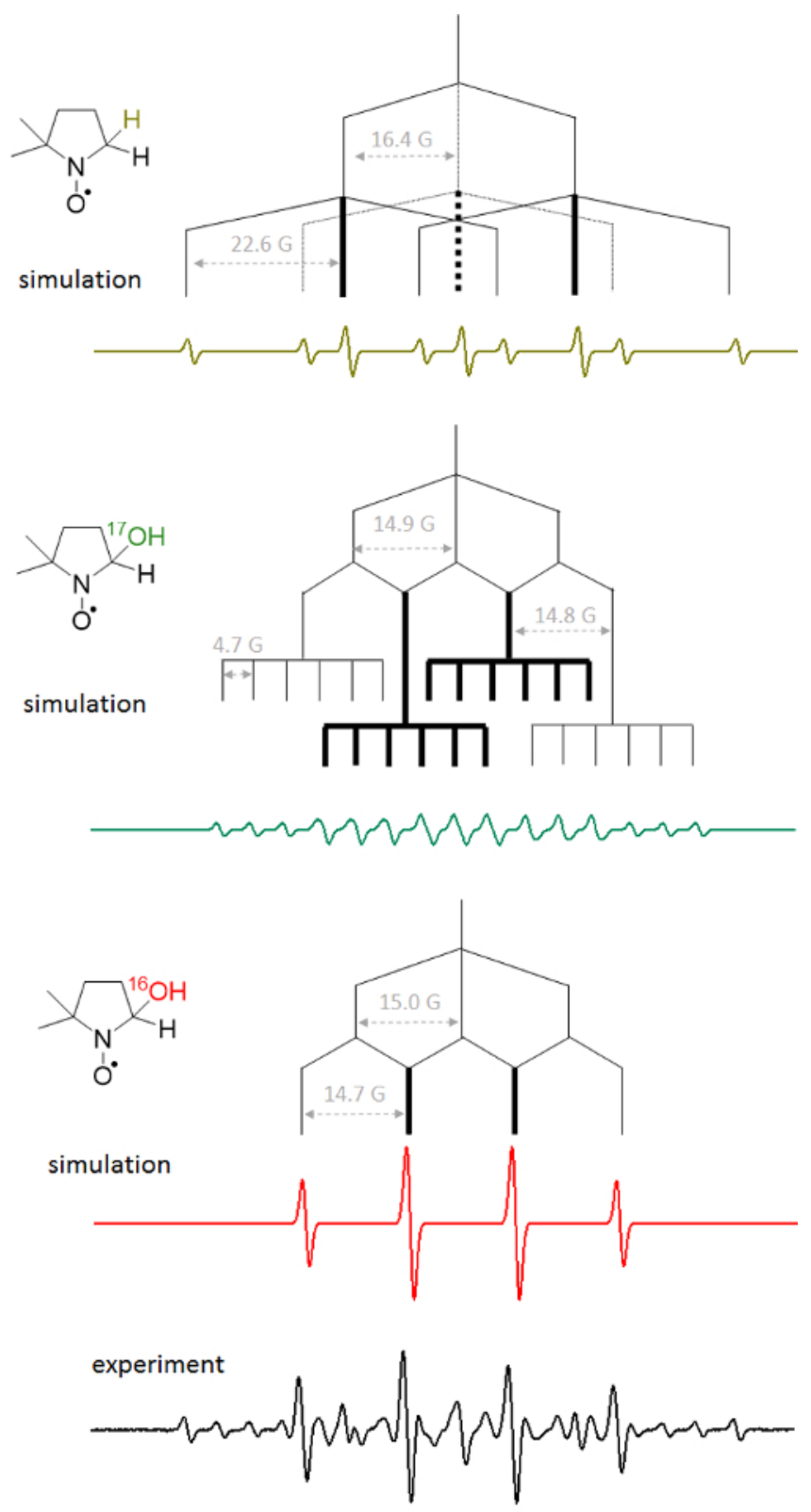

$\begin{array}{lllll}3460 & 3480 & 3500 & 3520 & 3540\end{array}$

Field / G

Figure 2. An EPR spectrum of the mixture of DMPO-H, DMPO- ${ }^{16} \mathrm{OH}$ and DMPO- ${ }^{17} \mathrm{OH}$ radical adducts induced in the solution of DMPO in $\mathrm{H}_{2}{ }^{17} \mathrm{O}$ exposed to plasma. The analysis was performed using the spectra simulation software using the hyperfine values available in literature ${ }^{16}$. Please click here to view a larger version of this figure. 


\begin{tabular}{|c|c|c|c|c|}
\hline \multirow{2}{*}{ Entry } & \multirow{2}{*}{$\begin{array}{c}\text { Relative humidity } \\
\left(\mathrm{H}_{2}{ }^{16} \mathrm{O}\right) \text { of the feed } \\
\text { gas, } \%\end{array}$} & \multicolumn{2}{|c|}{ Adduct concentration, $\mu \mathrm{M}$} & \multirow{2}{*}{$\begin{array}{c}\mathrm{H}_{2}{ }^{16} \mathrm{O} \text { delivered to } \\
\text { the liquid } \mathrm{H}_{2}{ }^{17} \mathrm{O} \\
\text { sample, } \%\end{array}$} \\
\hline & &.${ }^{17} \mathrm{OH}$ &.${ }^{16} \mathrm{OH}$ & \\
\hline 1 & 0 & 6 & 5 & 19 \\
\hline 2 & 10 & 8 & 18 & 25 \\
\hline
\end{tabular}

Table 1. Concentration of the DMPO- ${ }^{16} \mathrm{OH}$ and DMPO- ${ }^{17} \mathrm{OH}$ radical adducts and the amount of $\mathrm{H}_{2}{ }^{16} \mathrm{O}$ in the liquid $\mathrm{H}_{2}{ }^{17} \mathrm{O}$ sample after the plasma exposure. The absolute quantities of adducts concentrations were obtained using the EPR calibration with the stable radical 2,2,6,6Tetramethylpiperidine 1-oxyl (TEMPO). In the case of no added water vapor (entry 1), a residual humidity was present in the feed gas. The relative amounts of $\mathrm{H}_{2}{ }^{17} \mathrm{O}$ and $\mathrm{H}_{2}{ }^{16} \mathrm{O}$ in the liquid sample were determined using a hydrolysis reaction of cinnamoyl chloride yielding a mixture ${ }^{16} \mathrm{O}$ - and ${ }^{17} \mathrm{O}$-cinnamic acids upon reaction with the post-plasma exposure solution. The resulting mixture was analyzed by high resolution mass spectrometry as described elsewhere ${ }^{15}$.

\section{Discussion}

Here, we demonstrate the use of an in-house built atmospheric pressure plasma setup. The metal mesh cage helps to achieve reproducible plasma conditions with minimized interference from external fields, at the same time protecting nearby sensitive equipment from possible interference and/or damage by any plasma-induced fields. The shielding (caging) of the setup depends on the type of operated plasma and its electrical characteristics. The aim is to ensure the absence of external interference on the plasma operation and avoid the plasma fields interfering with surrounding equipment. In this case the mesh size is $22 \mathrm{~mm}$, however, reduced mesh size may be required for different plasmas. The plasma operation parameters were controlled using a voltage and a current probe connected to an oscilloscope. The introduction of the high voltage probe significantly changes the electric environment, and therefore the probe must become part of the electrical system and be positioned the same way throughout all of the experiments.

The use of the glass reactor encapsulating the sample and the plasma jet allows exclusion of the ambient atmosphere of often unknown composition from the reaction system. In the presented results (vide supra), it was used to determine the source of the plasma-induced reactive oxygen species in the aqueous sample exposed to the plasma effluent. Such investigation is possible if the molecules of the liquid water and the water in the feed gas (vapor) can be differentiated. To determine whether the hydroxyl radicals were formed in the gas phase or from the liquid water molecules, isotopically labelled water was introduced: $\mathrm{H}_{2}{ }^{17} \mathrm{O}$ as the liquid medium, $\mathrm{H}_{2}{ }^{16} \mathrm{O}$ vapor in the feed gas. If a hypothetical experiment was conducted in an open atmosphere, distinguishing between the two phases would have been hampered by the presence of the water vapor in the surrounding air. An alternative method to minimize the influence of the surrounding atmosphere was demonstrated in the literature, in which the diffusion of the species from the atmosphere into the plasma effluent was prevented using a shielding gas ${ }^{17}$. The shielding gas $\left(\mathrm{N}_{2}\right.$ or $\left.\mathrm{O}_{2}\right)$ creates a gas curtain with a known composition ${ }^{18}$. The reactor presented in this manuscript is a simple way to remove the influence of the ambient air components (such as water vapor), and can be used with different plasma jets without the introduction of the additional gas flow. Similar to the $\bullet \mathrm{OH}$ radical, the source of the $\bullet H$ radical can be determined by employing a $\mathrm{D}_{2} \mathrm{O} / \mathrm{H}_{2} \mathrm{O}$ system. The inexpensive $\mathrm{D}_{2} \mathrm{O}$ can also be introduced into the feed gas as a vapor as described above.

The saturation of the gas with $\mathrm{H}_{2} \mathrm{O}$ vapor was determined by weighing the Drechsel flask before and after bubbling the gas flow through it. The relative humidity (i.e., saturation) of the gas is calculated by the amount of the water evaporated and the volume of gas passed through.

Note that in prolonged experiments, the temperature of the liquid in the Drechsel flask may decrease due to the evaporation. The relative humidity is calculated for a specific temperature. The calculated values are further compared with those in literature ${ }^{19}$ to determine the relative humidity of the feed gas. We have empirically discovered that a flow of up to $2 \mathrm{slm}$ of He through a water-filled Drechsel flask fully saturates the gas with water vapor. However, elevated flow rates may not allow sufficient residence time of the gas in the liquid for full saturation. Other saturation techniques may be required.

Another challenging task is ensuring that no surrounding air is present in the system. The reactor is pre-flushed with the feed gas to remove the residual air. The time required for pre-flushing will depend on the volume of the reactor and the flow of the feed gas. The absence of external ambient air diffusion and entrainment into the system such as a helium feed gas plasma system can be tested using an $\bullet$ NO radical trapping reaction. Nitric oxide generated by plasma from $\mathrm{N}_{2}$ and $\mathrm{O}_{2}$ molecules of air can be detected by EPR as a radical adduct of the (MGD) ${ }_{2} \mathrm{Fe}{ }^{2+}$ complex $^{20}$ (MGD = N-methyl-D-glucamine dithiocarbamate). In the case of the complete absence of air, the EPR signal of the adduct is not observed. The absence of external water molecules in the reactor can be demonstrated by the following experiment. $A$ liquid sample of $D_{2} O$ is exposed to a dry feed gas plasma. The NMR analysis of the post-exposure sample reveals the amount of $\mathrm{H}_{2} \mathrm{O}$ brought into the liquid during the exposure. This allows to estimate the amount of the residual $\mathrm{H}_{2} \mathrm{O}$ in the tubing used for the feed gas ${ }^{15}$ in the experiment.

The sample container design is crucial in the experimental work. Initially, we have attempted using plastic and glass microcentrifuge tubes. Together with relatively high plasma feed gas flow, the small diameter of the opening does not let the surrounding air penetrate the microcentrifuge tube. However, this has many disadvantages. The plasma exhibited arching and large temperature increase near the edges of the microcentrifuge tube. The delivery of the species from the gas phase into the liquid was also significantly less efficient due to the different gas phase dynamics and the low surface area (and large volume) of the liquid sample. Thus, the surface area of the liquid sample is crucial for the delivery of the reactive species from the gas phase to the liquid sample. This is especially important for the short-lived radicals. The liquid sample container must therefore be designed to allow the exposed liquid to have high surface area for efficient diffusion. The sample should also have low depth to minimize the convection-related limitations of the liquid sample. It must be taken into account that elevated gas flows and especially with ignited plasma create significant disturbances at the surface of the liquid sample ${ }^{21}$. Therefore, the sample container has a welllike shape with diameter and depth required for the specific experiment. The height of the stand on which the well is positioned can be adjusted to experimental needs. The rubber grommet through which the plasma jet is inserted into the reactor makes it possible to change the contact angle of the effluent with the liquid. 
The presented method allows investigation of the source of the reactive species $(\cdot \mathrm{OH}, \cdot \mathrm{H}$, etc.) induced in the liquid by a $\mathrm{kHz}$ frequency parallel field plasma jet. The method employing a glass reactor surrounding the jet is not limited to the described conditions, and can be used with other atmospheric pressure plasmas. The method allows introduction of any admixtures to the feed gas: vapor, $\mathrm{O}_{2}, \mathrm{~N}_{2}$, etc. Among its other advantages is the possibility of conducting optical measurements inside it, although in this case optical quality quartz glass must be used as a reactor material. The exhaust tube in the lower part of the reactor allows using the plasma jet in virtually any lab: the exhaust can be connected via plastic tubing to a remote extraction hood. The reactor concept is versatile and can be used in research of different plasmas where controlled atmosphere is required. For example, polymerization of styrene is inhibited by the oxygen species ${ }^{22}$, but can be observed in the reactor when liquid styrene is exposed to the helium feed gas plasma.

\section{Disclosures}

The authors have nothing to disclose.

\section{Acknowledgements}

The authors thank Chris Mortimer, Chris Rhodes (Department of Chemistry workshops) and Kari Niemi (York Plasma Institute) for their help with the equipment. The work was supported by the Leverhulme Trust (grant No. RPG-2013-079) and EPSRC (EP/H003797/1 \& EP/K018388/1).

\section{References}

1. Boxhammer, V., et al. Bactericidal action of cold atmospheric plasma in solution. New J. Phys. 14, 113042 (2012).

2. Graves, D. B. The emerging role of reactive oxygen and nitrogen species in redox biology and some implications for plasma applications to medicine and biology. J. Phys. D: Appl. Phys. 45, 263001 (2012).

3. von Woedtke, T., Reuter, S., Masur, K., Weltmann, K.-D. Plasmas for medicine. Phys. Rep. 530, 291-320 (2013).

4. Machala, Z., et al. Formation of ROS and RNS in Water Electro-Sprayed through Transient Spark Discharge in Air and their Bactericidal Effects. Plasma Proc. Polym. 10, 649-659 (2013).

5. Lu, X., et al. Reactive species in non-equilibrium atmospheric-pressure plasmas: Generation, transport, and biological effects. Phys. Rep. 630, 1-84 (2016).

6. Takamatsu, T., et al. Microbial Inactivation in the Liquid Phase Induced by Multigas Plasma Jet. PLoS One. 10, e0132381 (2015).

7. Ahlfeld, B., et al. Inactivation of a Foodborne Norovirus Outbreak Strain with Nonthermal Atmospheric Pressure Plasma. mBio. 6, 02300 (2015).

8. Hirst, A., et al. Low-temperature plasma treatment induces DNA damage leading to necrotic cell death in primary prostate epithelial cells. Brit. J. Cancer. 112, 1536-1545 (2015).

9. Norberg, S. A., Tian, W., Johnsen, E., Kushner, M. J. Atmospheric pressure plasma jets interacting with liquid covered tissue: touching and not-touching the liquid. J. Phys. D: Appl. Phys. 47, 475203 (2014).

10. Greb, A., Niemi, K., O'Connell, D., Gans, T., Energy resolved actinometry for simultaneous measurement of atomic oxygen densities and local mean electron energies in radio-frequency driven plasmas. Appl. Phys. Lett. 23, 234105 (2014).

11. Wagenaars, E., Gans, T., O'Connell, D., Niemi, K., Two-photon absorption laser-induced fluorescence measurements of atomic nitrogen in a radio-frequency atmospheric-pressure plasma jet. Plasma Sources Sci. Technol. 21, 042002 (2012).

12. Abd-Allah, Z., et al. Mass spectrometric observations of the ionic species in a double dielectric barrier discharge operating in nitrogen. $J$. Phys. D: Appl. Phys. 48, 085202 (2015).

13. Takamatsu, T., et al. Investigation of reactive species using various gas plasmas. RSC Adv. 4, 39901-39905 (2014).

14. Uchiyama, H., et al. EPR-Spin Trapping and Flow Cytometric Studies of Free Radicals Generated Using Cold Atmospheric Argon Plasma and X-Ray Irradiation in Aqueous Solutions and Intracellular Milieu. PloS One. 10, 0136956 (2015).

15. Gorbanev, Y., O'Connell, D., Chechik, V. Non-thermal plasma in contact with water: The origin of species. Chem. Eur. J. 22, 3496 -3505 (2016).

16. Spin Trap Database, National Institute of Environmental Health Sciences. Available online at: http://tools.niehs.nih.gov/stdb/ accessed 05.04 . (2016).

17. Schmidt-Bleker, A., Winter, J., Iseni, S., Rueter, S. Reactive species output of a plasma jet with a shielding gas device - Combination of FTIR absorption spectroscopy and gas phase modelling. J. Phys. D: Appl. Phys. 47, 145201 (2014).

18. Schmidt-Bleker, A., et al. On the plasma chemistry of a cold atmospheric argon plasma jet with shielding gas device. Plasma Sources Sci. Technol. 25, 015005 (2015).

19. Lide, D. R (Ed.), CRC Handbook of Chemistry and Physics. CRC Press, Boca Raton, Florida, USA (1992).

20. Tsuchiya, K., et al. Nitric oxide-forming reactions of the water-soluble nitric oxide spin-trapping agent, MGD. Free Radic. Biol. Med. 27, 347-345 (1999).

21. Robert, E., et al. Rare gas flow structuration in plasma jet experiments. Plasma Sources Sci. Technol. 23, 012003 (2014).

22. Allen, T. L. Oxygen inhibition of the polymerization of styrene. J. Appl. Chem. 4, 289-290 (1954). 